\title{
Imaginación, creatividad y fantasía en Lev S. Vygotski: una aproximación a su enfoque sociocultural
}

\author{
Imagination, Creativity and Fantasy in Lev S. Vygotski: \\ An approximation to his sociocultural approach
}

\author{
Nicolás Alessandroni ${ }^{1}$ \\ Consejo Nacional de Investigaciones Científicas y Técnicas (CONICET) \\ Universidad Nacional de La Plata (UNLP), Argentina
}

\begin{abstract}
Resumen. Este trabajo persigue dos objetivos; en primer lugar, exponer sucintamente algunas de las características de las categorías conceptuales que constituyen el núcleo duro de los enfoques socioculturales, tanto en su formulación vygotskiana (Teoría Histórico-Cultural), como en aquellas postvygotskianas. Ello permitirá sentar bases conceptuales desde las cuales encarar el segundo objetivo, a saber, analizar el texto Imaginación y creatividad en el adolescente [Pedologija podrostka (Pedagogía del Adolescente), 1931] de Lev S. Vygotski. Dicho texto resulta un caso de análisis útil tanto para ilustrar el abordaje sociocultural del autor en relación con la creatividad y su vinculación con otras funciones psicológicas al interior del desarrollo sociogenético del individuo, como su poder de creación de consciencia y control autorregulatorio. las organizaciones y la sociedad en reducir las desigualdades en el lugar de trabajo y apoyar mejores acciones preventivas.
\end{abstract}

Palabras clave. Teoría histórico-cultural y enfoques socioculturales, Vygotski, imaginación, creatividad, Psicología del Desarrollo.

Abstract. This article has two aims. First, to expose briefly some characteristics of the key conceptual categories of sociocultural approaches, both in its Vygotskian (Historical-Cultural Theory) and post-Vygotskian interpretations. This will lay the conceptual foundations needed to address the second aim, that is, to analyse the text Imagination and Creativity in the Adolescent [Pedologija podrostka (Pedagogy of the Adolescent), 1931] by Lev S. Vygotski. This text makes up a useful case to explain the author's sociocultural approach about creativity and its relationship with other psychological functions within the sociogenetic development of subjects, as well as its power of creation of consciousness and autoregulatory control.

Keywords. Historical-cultural theory \& sociocultural approaches, Vygotski, imagination, creativity, developmental psychology.

\footnotetext{
${ }^{1}$ Nicolás Alessandroni. Instituto de Investigaciones Filosóficas (SADAF) - Consejo Nacional de Investigaciones Científicas y Técnicas (CONICET). Universidad Nacional de La Plata (UNLP), Argentina. Dirección postal: Bulnes 642, C.A.B.A (C1176ABL), Argentina.. E-mail: n.alessandroni@conicet.gov.ar
} 


\section{Las categorías conceptuales de los enfoques socioculturales}

Siguiendo la lectura que Baquero (1996) hace de Vygotski como matriz interpretativa, en esta primera sección se explorararán algunas categorías importantes al interior de la Teoría Histórico-Cultural, tal como fuera planteada inicialmente por el autor ruso y recepcionada posteriormente por otros teóricos. Para ello, se hará pausa, especialmente, en diversos aspectos básicos del núcleo duro de la teoría vinculados con:

1. El origen histórico y social de los Procesos Psicológicos Superiores (PPS). La diferenciación entre Procesos Psicológicos Elementales (PPE) y Superiores Rudimentarios (PPSr) y Avanzados (PPSa).

2. El papel central que cumplen los instrumentos de mediación en la constitución de los PPS. Los aportes de los teóricos de la actividad.

3. El necesario abordaje genético y no teleológico de los PPS. La idea de desarrollo psicológico como reorganización de funciones psíquicas o revolución psíquica de la consciencia. Las características de las unidades de análisis en este paradigma.

Con este análisis se espera circunscribir, de modo general, el alcance de algunas categorías analíticas que pueden ser heurísticos útiles a la hora de analizar el tratamiento de Vygotski a la creatividad durante la adolescencia como fase transicional del desarrollo, cuestión que se abordará en la segunda sección del escrito.

\section{Definición de Procesos Psicológicos Superiores}

En la Teoría Histórico-Cultural, el concepto de PPS hace referencia a ciertas funciones psicológicas específicamente humanas que tienen origen en -y pertenecen al dominio de- la vida social; están caracterizadas por poseer (o haber poseído en su historia reconstructiva) algún tipo de control consciente; además, se valen de instrumentos de mediación. Así, por ejemplo, el lenguaje se erige como uno de los PPS más importantes del desarrollo psicológico. Los procesos pertenecientes a este dominio históricosocial, si bien son ontogenéticamente posteriores a los PPE, no constituyen formas evolucionadas de estos últimos. Más bien, son el resultado de procesos de reorganización psíquica que poseen una legalidad diferenciada (Baquero, 1996) de aquella que caracteriza a los procesos evolutivos biológicos. Esto es, los PPS no constituyen una continuación lineal de otros procesos de nivel básico, sino que requieren de la apelación a un nivel cultural para ser comprendidos cabalmente.

Se dijo que los PPS dependen, en algún sentido, del dominio histórico-cultural. Sin embargo, esta relación de dependencia no debe ser confundida con un vínculo causal en términos de variable independiente-variable dependiente. Al contrario, desde la Teoría HistóricoCultural se sostiene que la vida cultural no modula el desarrollo de procesos superiores, sino que los genera a partir de un proceso de desarrollo diferenciado. Dicho de otro modo, el vínculo entre cultura y procesos psicológicos es bidireccional y de co-determinación; en fin, dialéctico. Es en este sentido que es posible afirmar, por ejemplo, que al tiempo en que un sujeto se apropia de la cultura, la cultura se apropia del sujeto.

Este tipo de abordajes holísticos no se restringen a la propuesta de Vygotski. Todo el campo de la Psicología Cultural actual propone, en línea con lo planteado supra, incluir como elemento clave a la cultura y a su relación con el individuo como motor del desarrollo cognoscitivo (Valsiner, 2000, 2007, 2012). Mente y cultura deben ser entendidas como dos términos indisociables de un proceso de co-construcción en el que ambas están en la génesis y son producto de la otra (Cole, 1996/1999; Cubero Pérez \& Santamaría Santiagosa, 2005; Wertsch, 1998).

Como se desprende de lo dicho, en este paradigma el origen del psiquismo es social y está garantizado por la participación del sujeto en actividades compartidas con otros sociales, en el marco de procesos culturales organizados en un contexto activo. Conviene, en este punto, precisar la noción de contexto, ya que con ella se designa no solo a la especificación de las circunstancias en las que tiene lugar el pensamiento, sino también a aquello que rodea y entrelaza al funcionamiento psíquico, a "una relación cualitativa entre un mínimo de dos entidades analíticas que son dos momentos en un único proceso" (Cole, 1996/1999, p. 129), o a 
una "envoltura supraindividual con respecto a la cual se definen el objeto/ambiente, el texto/contexto" (Cole, 1996/1999, p. 135). Desde esta perspectiva, el funcionamiento intersubjetivo y las prácticas culturales no se piensan como "incidentes, sino como inherentes al propio desarrollo y aprendizaje" (Baquero, 2001a, p. 165).

\section{Diferenciación entre PPSry PPSa}

Los PPS, tal como se definió en el apartado anterior, se instancian en dos tipos de procesos distintos: rudimentarios y avanzados. La diferencia entre ambos tipos de procesos radica en el prototipo de prácticas socioculturales que están a la base de ellos y en el tipo de uso de herramientas de mediación que los caracteriza. Así, los PPSr son específicamente humanos, pero emergen como resultado de las prácticas sociales genéricas de la especie humana; es decir, de aquellos procesos que habilitan la socialización primaria, razón por la cual son poseídos por todos los individuos humanos.

A diferencia de ellos, los PPSa requieren de prácticas sociales diseñadas específicamente para su transmisión (por ejemplo, contextos de enseñanzaaprendizaje escolares), involucran un mayor uso de instrumentos de mediación, poseen un mayor grado de independencia del contexto y también de regulación voluntaria. La distinción entre estos tipos de procesos superiores opera al interior del dominio sociocultural; en palabras de Baquero (1996):

La distinción entre PP Elementales y PP Superiores se situaría en la diferenciación y transición entre el dominio filogenético y el socio-cultural, mientras que la discriminación entre Procesos Rudimentarios y Avanzados, que operaría en el interior de los Procesos Superiores, supone un tratamiento de las transiciones genética dentro del dominio sociocultural (p. 35).

Resulta ilustrativo complementar esta distinción vygotskiana entre procesos superiores con la propuesta cartográfica de Ángel Rivière respecto de las funciones mentales (Rivière, 2002), que en su modelo se corresponde con las funciones Tipo III y Tipo IV. Las funciones Tipo III (que en la jerga vygotskiana se llamarían superiores rudimentarias) poseen un componente de habilitación biológica (están permitidas por el genoma, dice Rivière) y surgen en todas las sociedades humanas gracias a la existencia de ciertos tipos de contextos interactivos particulares, como aquellos propios de las situaciones de crianza. El lenguaje, en este modelo, corresponde a una función de Tipo III. Estos contextos interactivos, nota el autor, no están diseñados con el objetivo de promover las funciones que finalmente promueven. Esto es, las pautas de interacción que operan en interior (como aquellas que caracterizan a la relación madre-bebé) no son planificadas deliberadamente $y$, sin embargo, logran promover las funciones Tipo III con un alto nivel de eficacia.

Las funciones Tipo IV (vinculadas con aquellas que Vygotski denominó superiores avanzadas) son dependientes de formas especializadas y artificiales de interacción que están "dirigidas a la interiorización por el niño de ingenios de la cultura" (Rivière, 2002, p. 216). En este sentido, si las funciones Tipo III humanizan (son aquellas que definen a la especie de manera ubicua), las funciones Tipo IV enculturan y, en este sentido, pueden no ser compartidas por todos los humanos. Así, es difícil concebir un humano con una trayectoria de desarrollo "típico" que no hable, pero no un humano que no pueda codificar los sonidos que escucha en una partitura recurriendo a símbolos específicos de la notación musical moderna.

Por otra parte, las funciones Tipo IV no se encuentran determinadas filogenéticamente (como sí lo están las funciones Tipo I y II y también las Tipo III, en menor medida), sino históricamente. Pertenecen al ámbito particular de la cultura de una sociedad y, es por ello que, además, son las funciones menos económicas. Estas obtienen resultados cognitivos prescindiendo de una economía de recursos (cuestión que no ocurre con las funciones Tipo I, que son muy eficaces; por ejemplo, el establecimiento visual de las constantes de brillo). En palabras de Rivière, son aquellas que presentan los mayores "problemas de interiorización" (Rivière, 2002, p. 220), porque son "como alianzas provisionales y altamente volátiles entre subsistemas, 
por eso el aprendizaje escolar viene y va y se pierde" (Rivière, 2002, p. 225).

Desde esta perspectiva, el ámbito escolar, por ejemplo, puede verse como el terreno concreto donde se despliegan prácticas particulares que favorecen el "impulso, desarrollo y complejización creciente de los Procesos Psicológicos Superiores" (Baquero, 1996, p. 99).

\section{En busca del mecanismo explicativo del surgimiento de los PPS}

Habiendo establecido que el origen de los Procesos Psicológicos Superiores debe buscarse en el dominio sociocultural, es menester, ahora, caracterizar al mecanismo que permite al sujeto aprehender y dominar las pautas de comportamiento sociales y las formas de uso de los instrumentos de mediación de su entorno. Este mecanismo está dado por lo que Vygotski denominó interiorización de las funciones psicológicas, a saber, la reconstrucción en el plano intrapsicológico de una función cuya forma primigenia debe buscarse en el ámbito interpsicológico.

Una lectura simplista de este mecanismo podría implicar que los sujetos, gracias a la interiorización, acumulan en sus mentes copias exactas de las funciones interpsicológicas. Sin embargo, Vygotski hace notar que el proceso de interiorización está caracterizado por una reorganización de la actividad psicológica original; es decir, por una operación de reconstrucción de la función, un cambio en su estructura.

En consonancia con la consideración del vínculo dialéctico y co-formativo entre mente y cultura que sostiene este paradigma, la interiorización de funciones psicológicas no debe conceptualizarse como la recepción en la conciencia de contenidos externos, sino como un proceso creador de consciencia (Baquero, 1996, p. 45) que implica una vinculación genética con ciertos instrumentos de mediación que poseen carácter también formativo. En palabras de Wertsch:

Existe una conexión íntima, enraizada en las transiciones genéticas, entre las estructuras específicas y los procesos de funcionamiento inter e intramental, los cuales, a su vez, implican que diferentes formas de funcionamiento intermental dan lugar a la emergencia de diferencias relativas en las formas de funcionamiento intramental" (Wertsch, 1991/1993, p. 27).

\section{Una concepción del desarrollo psicológico no teleológica}

A partir de lo desarrollado hasta aquí, es dable definir al desarrollo psicológico superior como el resultado del entrelazamiento de dos líneas del desarrollo (una natural y otra cultural), posibilitado por la participación en prácticas sociales específicas, en contextos particulares, y caracterizado por la apropiación de diferentes instrumentos de mediación y el dominio progresivamente adquirido sobre diferentes sistemas de representación. Sin embargo, conviene detenerse por unos instantes en la concepción vygotskiana de dirección del desarrollo psicológico.

Ya se ha adelantado que la psicología de Vygotski es de corte antinaturalista, por cuanto no reconoce que exista una continuidad lineal entre el desarrollo de los PPE y los PPS y, en cambio, hace depender a estos últimos de una legalidad específica del dominio sociocultural. En relación con este punto, y al contrario de lo sostenido por otros psicólogos del desarrollo (por ejemplo, Piaget, 1936/1981), Vygotski sostiene que el desarrollo psicológico funciona al modo de una integración dialéctica no teleonómica o de una reorganización del funcionamiento psicológico global, caracterizada por una variación de las interrelaciones funcionales entre los diversos procesos psicológicos.

Desde esta perspectiva, no existe una relación de necesidad lógica o antecedencia causal entre una función psicológica y otra, ni tampoco una estricta relación de superación cualitativa: el lenguaje escrito no constituye la forma avanzada del lenguaje oral y, como se verá en la segunda sección del trabajo, la creatividad del adulto no constituye una versión mejorada de la creatividad del adolescente. Lo que caracteriza la transición entre diferentes momentos evolutivos son los cambios en las relaciones interfuncionales del sistema psíquico todo, el cual tiene lugar bajo la forma de una compleja dinámica de interiorización-reorganización. Así, el desarrollo psíquico se caracteriza por presentar relaciones de 
convergencia y mutua reorganización de los procesos durante su curso.

\section{La función autorregulatoria de las funciones psicológicas}

Este proceso de interiorización de instrumentos y signos que circulan en el medio social hacia la esfera intrapsicológica convierte los sistemas de regulación externa en medios de regulación interna, es decir, en herramientas para el control y la regulación de las propias funciones psicológicas. De esta manera, la progresiva capacidad autorregulatoria del sujeto se inserta como hito evolutivo en el proceso de obtención de logros crecientes en el dominio de la naturaleza, la cultura y sobre sí mismo; es decir, en el curso del desarrollo psíquico entendido como apropiación de objetos, saberes, normas e instrumentos culturales en contextos de actividad conjunta socialmente definidos (Baquero, 1999).

La lógica de las condiciones de posibilidad de los mecanismos autorregulatorios debe buscarse en las implicancias de la participación del sujeto en prácticas sociales culturalmente determinadas. Tal como explica Rivière, "El contacto social con uno mismo se posibilita por el hecho de que la organización psicológica, a través de la relación con los demás, se desdobla y replica con respecto a sí misma, las pautas de inter-acción” (Rivière, 1988, p. 82).

Un caso de función psicológica autorregulatoria es el habla privada. En los párrafos que siguen se presentan brevemente, a modo de ejemplo, algunos desarrollos vygotskianos y posvygotskianos al respecto. El término habla privada refiere al habla exteriorizada y audible que, no estando dirigida a otra persona, cumple una función eminentemente autorregulatoria (Winsler, 2009).

$\mathrm{Al}$ contrario de lo planteado por Piaget, quien afirmaba que este tipo de manifestaciones debía entenderse como consecuencia de un egocentrismo cognitivo generalizado y de un lenguaje social pobre (Winsler, Díaz, \& Montero, 1997), fue Vygotsky quien propuso que el habla privada, en lugar de poseer un origen de tipo interno y una trayectoria de desarrollo ontogenético inside-out, encuentra sus condiciones de posibilidad en la esfera sociogenética, es decir, en el mundo social del infante, en la trama interactiva que el infante teje junto con otros, para luego ser interiorizado bajo la forma de herramienta para guiar el pensamiento y resolver problemas (Vygotsky, 1973/1987[1934]).

El habla privada representa, entonces, la apropiación subjetiva progresiva de la historia de interacciones sociales con otros. En este proceso de interiorización, la estructura del lenguaje sufre algunas modificaciones relativas; por ejemplo, a la longitud de las expresiones, a su claridad estructural y al carácter predicativo de ellas, lo que genera que el lenguaje se vuelva progresivamente más abreviado y se elidan los sujetos de la enunciación, prescindiendo así de la necesidad de actualización semiótica propia del lenguaje social.

En relación con la función del habla privada, Vygotsky argumenta que:

Según la concepción de Piaget, el lenguaje egocéntrico del niño no se adapta a la inteligencia de los adultos [...] El habla egocéntrica no cumple ninguna función en el pensamiento o la actividad realista del niño: simplemente los acompaña $[\ldots]$ Los resultados de nuestras experiencias indican que la función del lenguaje egocéntrico es similar a la del lenguaje interiorizado: no constituye un mero acompañamiento de la actividad del niño, sino que sirve de ayuda a la orientación mental [...] (Vygotsky, 1973/1987[1934], p. 174-175).

El lenguaje, en tanto herramienta cultural simbólica, es utilizado primero con fines comunicativos interpersonales $\mathrm{y}$, más tarde, es utilizado en la esfera intrapersonal con fines comunicativos y de autorregulación. Con la ayuda del habla privada es posible para los niños lograr una distancia respecto de los estímulos circundantes y del contexto inmediato para dar lugar a sus planes internos. Sin embargo, las posibilidades que brinda el habla privada no se restringen a la adquisición de cierta maestría sobre las características del entorno.

Existe evidencia para pensar que el habla privada facilita también la transición desde la performance colaborativa hasta la performance independiente. Además, por estar basada en la transferencia del rol 
regulatorio desde el adulto hasta el niño, su utilización por parte de los infantes es conducente al éxito en la planificación, realización y monitorización de tareas (Winsler, Díaz \& Montero, 1997).

El habla privada se erige así como mediador metacognitivo privilegiado de la función autorregulatoria durante el curso de actividades dirigidas a metas (Atencio \& Montero, 2009). Aún más, es conocido que la utilización del habla privada es, en los niños, directamente proporcional a la dificultad de la tarea a resolver (Fernyhough y Fradley, 2005; Berk, 1992).

Se plantea así la necesidad de considerar la relación entre habla privada y performance como un vínculo "dinámico y complejo en el que la dificultad de la tarea actúa como variable que interactúa con el habla privada, modulando sus efectos en la performance" (Montero \& De Dios, 2006, p. 176). A medida que una tarea se vuelve más dificultosa -es decir, desafiante para el nivel de desarrollo actual de competencia no mediada-, el niño utilizará el habla privada como un medio para ganar dominio en la tarea.

Además de funcionar como una herramienta para la modulación de los procesos cognitivos, se ha propuesto que el habla privada se encuentra vinculada con la transmisión de los procesos motivacionales humanos. En este sentido, se argumenta que, además de colaborar con la planificación y organización cognitiva general, el habla privada se encuentra ligada a la dimensión volitiva del self, al establecimiento de metas, a la auto-evaluación subjetiva de la performance enactiva y a la adecuación experiencial del contenido intencional de nuestras acciones, dimensiones que se encuentran en marcadas al interior de las categorías motivación intrínseca y motivación intrínseca. De esta forma,

Los individuos pueden socavar o mejorar su propia motivación intrínseca en relación con una tarea a partir de la emisión de expresiones de habla privada con valencias diferenciadas (Atencio \& Montero, 2009, p. 209).

Si bien hasta ahora solo se ha mencionado la utilización que realizan los niños del habla privada, es menester notar que los adultos también hacen uso de este recurso del pensamiento. Por ejemplo, se ha comprobado que, en contextos en los que se vuelve necesario alternar rápidamente entre dos o más tareas, recurrir al habla privada disminuye notablemente el costo de alternancia (Winsler, 2009). Esto sugeriría que, ante determinados conflictos o desafíos cognitivos, los adultos son capaces de re-externalizar (Fernyhough, 2004) el habla privada con fines de autorregulación y reorganización de la actividad mental.

El breve desarrollo realizado en relación con esta temática solo intentó destacar el modo en que ciertas herramientas semióticas "están disponibles y ordenan la vida social y, como resultado de su apropiación, podrán constituirse en herramientas de regulación del propio psiquismo" (Baquero, 2012a, p. 67).

\section{Los aportes de la Teoría de la Actividad}

Si se trata de evaluar las formas particulares que adopta el desarrollo psíquico en el tiempo, es posible considerar a la actividad como unidad de análisis y concepto bisagra entre las esferas individual y cultural (Baquero, 1996). Así, es posible pensar a la actividad como una interfaz que permite comprender los procesos de desarrollo cognitivo y cuyo análisis puede complementar la labor de aquellos teóricos que prefieren encarar el análisis sociocultural por la vía del análisis de la acción mediada.

La Teoría de la Actividad, tal como fuera enunciada por diferentes autores posvygotskianos, parte de considerar que el desarrollo psicológico se basa en actividades culturales prácticas. Esta noción de actividad que posee un antecedente etimológico en la acepción rusa del término praxis (Daniels, 2001/2003), involucra pensar a la mente individual dentro de ciertos sistemas de actividad. Fue Leontiev (1972/1977) quien planteó originalmente la distinción entre actividad, acción y operación. En sus desarrollos, la actividad es vista como una unidad molar y sistémica definida por un motivo. En tanto, la acción pertenece a un nivel inferior jerárquico y está determinada por sus fines. Por último, en el sustrato jerárquico inferior encontramos al concepto de operación, que es dependiente de una 
consideración de sus medios (Baquero, 1996, 2012b).

Posteriormente, Engeström (2015) sistematizó diferentes aportes vinculados con la Teoría de la Actividad, asiló tres generaciones de ella y destacó la necesidad de atender a los procesos de evolución de los sistemas de actividad y a su eventual adopción de formas institucionales y organizacionales. De este modo, y como un intento de solucionar el problema de la insensibilidad cultural de aportes previos sobre la actividad, situó en el centro de la escena a la relación entre los sistemas de actividad y ciertos elementos pertenecientes al macronivel de lo colectivo y de la comunidad, en vez de concentrarse en el individuo.

Además, Engeström postuló la urgencia de abordar el análisis de redes de sistemas de actividad en interacción, definidas dialógica y multivocalmente, y de las cuales pueden emerger conflictos. Para este abordaje, el autor propuso como unidad de análisis mínima al complejo compuesto por dos sistemas de actividad en interacción, cada uno de los cuales se define en términos de los siguientes subcomponentes: sujeto, instrumento, objeto, comunidad de referencia, reglas de la división de tareas y normas de la comunidad (Daniels, 2001/2003).

\section{Elproblema de las unidades de análisis}

Redefinir el desarrollo psíquico en relación con el medio sociohistórico y pensar el desarrollo humano como producto de la actividad intersubjetiva, culturalmente situada y mediada semióticamente (Baquero, 2001b), hace que la Teoría Histórico-Cultural sobrepase en su formulación teórica los límites de los procesos cognitivos individuales (Baquero, 2009). A su vez, hace que requiera como necesidad epistemológica del paradigma encontrar nuevas unidades de análisis que conserven las propiedades del todo sistémico, es decir, "vivas", para explicar los procesos de evolución de las funciones psicológicas (Baquero, 2012a).

Fue el mismo Vygotski quien avizó este problema al criticar las propuestas metodológicas de ciertas corrientes de pensamiento (por ejemplo, la Psicología Experimental) dedicadas a estudiar las funciones psicológicas desde lo que él llama un contexto reactivo.
Se trata de un marco de investigación que concibe al objeto de estudio como una entidad susceptible de ser escindida e interpelada apelando a constructos tales como factores o variables independientes y variables dependientes; es decir, constructos pertenecientes a la lógica estímulo-respuesta (Vygotski, 1979/2009 [19311934]). Retomando a Lewin (1931), caracterizó a este tipo de abordaje como un punto de vista fenotípico basado en la descripción de ciertos rasgos del fenómeno y la negación de la historia evolutiva del mismo.

En esta perspectiva, las totalidades complejas son analizadas a partir de la separación de lo que se considera son sus elementos constituyentes o componentes, método que Vygotski propone llamar de análisis por elementos. Es conocido el ejemplo brindado por Stuart Mill (2011 [1843]) para desacreditar este punto de vista: si se quisieran analizar las propiedades del agua, sería poco feliz proceder descomponiéndola en Hidrógeno y Oxígeno, puesto que ninguno de estos componentes posee las propiedades que sí posee el agua como compuesto (por caso, tanto el Hidrógeno como el Oxígeno alientan la permanencia y amplificación del fuego, mientras que el agua lo extingue).

Esta perspectiva resulta insuficiente para una Psicología que desee desentrañar los mecanismos explicativos de las funciones psicológicas, cuestión que había sido postulada también por Wundt en su Völkerpsychologie (1916/2003 [1904]), donde este último destacó la imposibilidad de estudiar experimentalmente las funciones psicológicas superiores ignorando la naturaleza unitaria y compleja que las define. En palabras de Vygotski, “(...) si la esencia de los objetos coincidiera con la forma de sus manifestaciones externas, la ciencia sería totalmente superflua" (Vygotski, 1979/2009 [1931-1934], p. 101-102).

Al contrario, Vygotski concibe a las funciones psicológicas al interior de la evolución psicológica del individuo y como parte del desarrollo dialécticohistórico general. Su compromiso marxista lo lleva a afirmar que el hombre debe ser estudiado en tanto modificador de la naturaleza y creador de nuevas condiciones de posibilidad para su existencia, sin perder de vista la organización de la estructura de la conciencia como una totalidad. De esta manera, un 
sistema psicológico no es una colección de elementos, sino un conjunto de procesos de tipo global que no pueden escindirse y deben enmarcarse siempre en condiciones de posibilidad históricas y culturales que permiten develar los nexos entre las dimensiones inter e intrasubjetivas que determinan el desarrollo de los sujetos. De allí que el "carácter histórico y cambiante de los sistemas psicológicos se expresa, a su vez, como la historia misma del desarrollo, que se constituye, al fin, en procesos graduales y también por saltos" (Castorina \& Baquero, 2005, p. 173).

El método que propone Vygotski es el método de análisis por unidades (Vygotski, 1973/1987[1934]), creado con el afán de no simplificar los fenómenos que se abordan y que busca tomar como unidad de análisis objetos complejos que conservan todas las propiedades básicas del total, ya que "no es cierto que una reacción compleja consista en una cadena de procesos separados que podrían añadirse y sustraerse arbitrariamente" (Vygotski, 1979/2009 [1931-1934], p. 107). Los procesos psicológicos, en esta visión, no deben ser entendidos como sumas algebraicas de componentes, tal como propone el método de análisis por elementos.

El método de análisis por unidades busca, en cambio, analizar procesos reconstruyendo cada uno de los estadios del desarrollo de los mismos para evitar ser engañados por lo que él denominó formas fosilizadas de conducta. Por ejemplo, muestras de conducta estáticas, analizadas transversalmente y despojadas de toda historia constructiva. Al contrario, el análisis debe ser dinámico, es decir, debe contemplar las íntimas relaciones entre presente y pasado ${ }^{1}$.

En sus propias investigaciones, Vygotski utiliza este método, por ejemplo, al estudiar las relaciones entre pensamiento y lenguaje, para lo cual postula como unidad de análisis compleja el significado de la palabra. Para el autor, la conexión entre pensamiento y palabra no es una vinculación primaria e invariante,

${ }^{1}$ De modo interesante, Cole (1996) ha propuesto integrar también la dimensión de futuro en el análisis de los procesos psicológicos, a través de, por ejemplo, el concepto de "prolepsis". sino que se origina en el desarrollo y se modifica y hace más estrecha a lo largo de él (Rivière, 1988). En este proceso de variación genética del significado, se exhiben las diferentes formas de relación entre pensamiento y lenguaje, funciones que tienen raíces genéticas distintas que, en el curso del desarrollo, se sintetizan dialécticamente.

De igual manera, al estudiar el desarrollo del habla privada, Vygotski se basa en la premisa de que ella "no suprime las formas de habla social o dialogada, sino que, por el contrario, se revierte sobre ellas o las co-define. En sentido estricto, los procesos intrae interpsicológicos se co-definen" (Castorina \& Baquero, 2005, p. 175), definiendo así a su objeto de estudio como una entidad inseparable (y en ciertos aspectos, indiferenciada) del contexto sociocultural en el que se enmarca. Tal como menciona Vygotski, estudiar algo históricamente implica estudiarlo en el proceso de su cambio y, estudiar el proceso de cambio de una función psicológica, implica considerar el "mundo de la cultura socialmente organizado, creado por un sujeto que, procesualmente, ha desarrollado sus fuerzas y habilidades" (Yaroshevsky, 1989, p. 19).

En sintonía con la propuesta vygotskiana y comprendiendo a la práctica pedagógica como el contexto social fundamental en el que tiene lugar la reproducción-producción cultural (Daniels, 2001/2003, p. 3), Baquero, por ejemplo, propone pensar como unidad de análisis a la educabilidad de los sujetos, ya no como una propiedad de los mismos, sino como "un efecto de la relación de las características subjetivas y su historia de desarrollo con las propiedades de una situación” (Baquero, 2001b).

Del mismo modo, Rogoff (1997) ha propuesto como unidad de análisis del desarrollo psicológico a la actividad (o evento), entendida esta como un sistema complejo que involucra tres planos (los del aprendizaje, la participación guiada y la apropiación participativa) y como constructo que permite reformular la relación entre el individuo y el desarrollo social y cultural, “(...) una relación en la que cada uno está implicado en la definición del otro. Ninguno de ellos existe separadamente" (Rogoff, 1997). 
¿Qué aporta el enfoque sociocultural de Vygotski al abordaje psicológico de la imaginación, la creatividad y la fantasía?

La imaginación, la creatividad y la fantasía forman parte del repertorio de las funciones psicológicas humanas, ocupando en él un lugar primordial. Diferentes estudios abonan esta afirmación y señalan, por ejemplo, que ellas resultan de importancia insoslayable tanto para la resolución de problemas de nivel individual (Maier, 1970) como para un amplio ámbito de actividades humanas sociales, como lo son el avance del conocimiento científico (Kim, 2016; Sánchez-Ruiz, Romo Santos \& Jiménez Jiménez, 2013), la innovación organizacional (Amabile, 1988) y la invención técnica y artística (Weisberg, 2006).

Dicho de otro modo, no caben dudas respecto de la utilidad cultural de la imaginación y la creatividad. Sin embargo, el estudio del desarrollo cultural de estas funciones psicológicas constituye, desde hace mucho tiempo, un ámbito de vacancia (Sternberg, 1999). En un artículo de 1983, por ejemplo, Amabile sostiene que "no existe investigación alguna acerca de la psicología social de la creatividad" (Amabile, 1983, p. 357).

Del mismo modo, Feldman, Csikszentmihalyi y Gardner (1994) resaltan las limitaciones del modelo hegemónico de investigación de la creatividad cuyos diseños metodológicos consisten en descripciones en profundidad de las vidas de individuos "descollantes" que no incluyen elementos culturales ni sociales.

En este modelo, la unidad de análisis es el individuo creativo que propulsa innovaciones por fuera (Paulus \& Nijstad, 2003) o a pesar de (Weiner, 2000) su contexto social. En palabras de Glăveanu (2010), en el modelo hegemónico, "la sociedad y la cultura actúan repetidamente como las 'villanas' contra las cuales lucha el creador" (p. 80).

La perspectiva sociocultural acerca de la creatividad del adolescente desarrollada por Vygotski resulta francamente opuesta al modelo individualista hegemónico. En ella, la sociedad y la cultura no son las villanas que obstaculizan los procesos de innovación, sino las condiciones de posibilidad de estos. Como se desarrollará en la próxima sección, para Vygotski la creatividad es un proceso psicológico superior cuyo origen ontogenético es histórico-cultural y está vinculado a contextos de actividad cotidianos donde se ponen en juego ciertos instrumentos de mediación semiótica.

Por este motivo, la unidad de análisis considerada por Vygotski excede la esfera individual y abarca la participación de los sujetos en sistemas de actividad culturalmente organizados. Además, la creatividad es abordada por el autor como una función psicológica en despliegue dinámico y esto le permite explicar, desde una óptica longitudinal del desarrollo humano, de qué modo pensamiento y creatividad se enlazan durante la adolescencia para nutrirse mutuamente y dar lugar a la emergencia de la novedad. Así, la creatividad es analizada como una función en desarrollo que se orienta desde la interacción social hacia la autorregulación y que se vincula tanto con los procesos cognitivos como con los aspectos afectivos de la vida psicológica de los sujetos.

\section{Un abordaje sociocultural de la imaginación, la creatividad y la fantasía}

"Es para uno mismo, en la mente, que se producen los poemas y las novelas, que se actúan los dramas y las tragedias, y que se componen las elegías y los sonetos" (Vygotski, 1998 [1931], p. 165).

Esta sección tiene el objetivo de analizar, en tanto caso ilustrativo, la propuesta vygotskiana para comprender la creatividad del adolescente. Para esta tarea, se hará uso de las categorías de análisis desarrolladas con anterioridad.

En Imaginación y creatividad en el adolescente [Pedologija podrostka (Pedagogía del Adolescente), 1931], Vygotski comienza por situar a la creatividad, la fantasía y la imaginación ${ }^{2}$ como funciones psicológicas superiores de relevancia insoslayable en el desarrollo del sujeto, porque lo habilitan tanto a interpretar y construir novedades, como a "transponer propiedades de las cosas, reemplazar unas por otras y combinar propiedades y acciones" (Vygotski, 1998 [1931], p. 
152-153). La consideración sobre la relevancia de estas funciones se refuerza, en el texto, a partir de la cita de ciertos testimonios clínicos de E. Cassirer (1929) respecto de pacientes con disturbios complejos de los PPS atendidos en un instituto neurológico de Frankfurt. Estos pacientes que, según Vygotski, carecían de fantasía y creatividad, no podían, entre otras cuestiones, repetir una frase cuyo contenido semántico no fuera congruente con la experiencia sensorial concreta individual. Del mismo modo, estos pacientes eran incapaces de utilizar objetos de la vida cotidiana en circunstancias no usuales.

Del análisis de estas viñetas clínicas, Vygotski extrae la conclusión de que la imaginación y la creatividad, caracterizadas por poder procesar libremente los elementos de la experiencia, requieren a la libertad interna de pensamiento, acción y cognición como precursora. Esta característica solo es alcanzada por aquellos sujetos que han logrado formar verdaderos conceptos.

Uno de los puntos más interesantes del escrito es la discusión que entabla Vygotski con la perspectiva tradicional en Psicología acerca de la creatividad, que la entiende como una función primordial que conduce el desarrollo mental del adolescente. Así, en este paradigma, la imaginación sería aquella función que caracteriza toda la vida intelectual del adolescente:

El punto de vista tradicional intenta subordinar todos los otros factores del comportamiento del adolescente a esta función básica, asumiendo que se trata de una manifestación primaria e independiente de los factores fundamentales y dominantes de toda la psicología de la maduración sexual (Vygotski, 1998 [1931], p. 153).

Por otra parte, la perspectiva tradicional ha obviado, según el autor, las relaciones entre la creatividad y la vida intelectual, solo considerando los vínculos de la misma con la esfera emocional. En contra de esta visión, Vygotski avanza una hipótesis: durante la adolescencia,

${ }^{2}$ En ciertos pasajes del manuscrito, estas tres funciones parecen utilizarse de modo intercambiable. En otros, Vygotski pareciera situar a la creatividad y a la imaginación como sub-tipos de fantasía. La resolución de esta precisión no reviste mayor importancia para el desarrollo argumental del autor. En cualquier caso, importa establecer que se trata de funciones estrechamente vinculadas. las funciones de la imaginación y el pensamiento en conceptos atravesarían procesos de reorganización psíquica que les permitirían conectarse. De esta forma, la imaginación en contacto con los conceptos se intelectualizaría y el pensamiento en conceptos -en contacto con la imaginación- se creativizaría. Esta relación de entrelazamiento inaugura una función totalmente nueva al interior de la personalidad del adolescente. La fantasía adolescente no sería, así, una función primaria y elemental, sino el resultado de la reestructuración psíquica de la creatividad infantil en contacto con la función de formación de conceptos.

Aun cuando Vygotski plantea esta alternativa hipotética sustantiva, debe dar, también, respuesta a emprendimientos investigativos empíricos. Estos indican que la utilización de imágenes eidéticas (es decir, representaciones visuales) que constituyen la expresión más notable de la fantasía infantil desaparece durante la adolescencia. Dicha cuestión parecería abonar la idea de que el pensamiento visual, al quedar despojado de sus funciones intelectuales, desaparecería para dar lugar al pensamiento lógico abstracto.

En este punto, la propuesta del autor es que, durante la adolescencia, la creatividad se libera de lo concreto, de los factores gráficos, pudiendo penetrar en el campo de los elementos abstractos. De esta forma, la creatividad empezaría a depender de los conceptos. No obstante, esta nueva vinculación no implica que la creatividad sea absorbida por el pensamiento lógico, sino que estas dos funciones se acercan dialécticamente la una a la otra, sin llegar nunca a fundirse. En función de ello, el autor afirma que: "Tenemos frente a nosotros el problema de encontrar las relaciones únicas entre los factores abstracto y concreto que son característicos de la imaginación durante la edad transicional" (Vygotski, 1998 [1931], p. 157).

Sería imposible hacer justicia al detallado análisis que inicia Vygotski en el tercer apartado del manuscrito para iniciar la vía de resolución de este problema de conocimiento. Sin embargo, interesa remarcar que, haciendo gala de su concepción del desarrollo psíquico como conjunto complejo de reorganizaciones dialécticas, el autor propone que la 
imaginación del adolescente posee sus bases genéticas en el juego del niño.

La única diferencia entre el juego concreto y la imaginación es la necesidad de recurrir al sustrato material: en lugar de jugar, el adolescente fantasea haciendo uso de representaciones visuales y, en vez de construir castillos en la arena, los construye en el aire (vale decir, en su mente). De esta manera, Vygotski rechaza que el pensamiento visual desaparezca junto con el advenimiento de la adolescencia; más bien, el pensamiento visual es "movido a otro lugar, a la esfera de la fantasía y cambia parcialmente bajo la influencia del pensamiento abstracto, elevándose, como todas las demás funciones, a un nivel más elevado" (Vygotski, 1998 [1931], p. 159). La formación de conceptos posibilita a la imaginación adolescente al permitirle liberarse de situaciones concretas y acceder a procesos de procesamiento creativo diferenciales (ver figura 1).

Ahora, resta explicar qué novedad emerge de este entrelazamiento dialéctico entre la imaginación adolescente (que es continuación del juego infantil) y el pensamiento en conceptos. Uno de los puntos de desacuerdo de Vygotski con otras teorías psicológicas radica en la aseveración de que el pensamiento abstracto se mueve en los planos abstracto y general, y la fantasía y la imaginación solo en el plano de lo concreto.

El autor entenderá, asimismo, que la imaginación es una actividad creativa que procede de lo concreto a lo concreto, pero dirá que dicho proceder es posible únicamente gracias a la ayuda de elementos abstractos.
Así, un proceso de fantaseo adolescente iniciaría con la consideración de una imagen visual que solo gracias al auxilio de verdaderos conceptos logra mutar en una nueva representación imaginada. Por otra parte, según el autor, la fantasía del adolescente es más creativa que la fantasía del niño; esta modificación cualitativa está íntimamente vinculada con los cambios en las condiciones de vida del adolescente. Durante la adolescencia, los sujetos atraviesan una revolución creativa, suelen exhibir una tendencia hacia la productividad y hacia la creación artística bajo la forma de diarios íntimos y poemas. Las imágenes que la imaginación adolescente utiliza quedan definidas, según el autor, por poseer un tono emocional.

No por ello menos importante, la fantasía del adolescente es consciente, lo cual la habilita, como a otros PPS, a cumplir funciones de autorregulación. La función autorregulatoria más importante de la fantasía, la imaginación y la creatividad está vinculada a la vida emocional. La fantasía resulta reparatoria allí donde existen necesidades, actitudes y sentimientos que perturban al adolescente $y$, por ello, es un "cumplimiento de deseo ${ }^{3}$, un correctivo de actividades que no brindan satisfacción (...) La vida que no se vive exteriormente encuentra expresión en las imágenes creativas" (Vygotski, 1998 [1931], p. 164-165).

Aún más, este uso autorregulatorio de la fantasía es privado: el adolescente no suele compartir sus fantasías con nadie, sino más bien todo lo contrario, es decir, las oculta. De esta forma, y por la vinculación ya establecida por el autor entre la imaginación y el

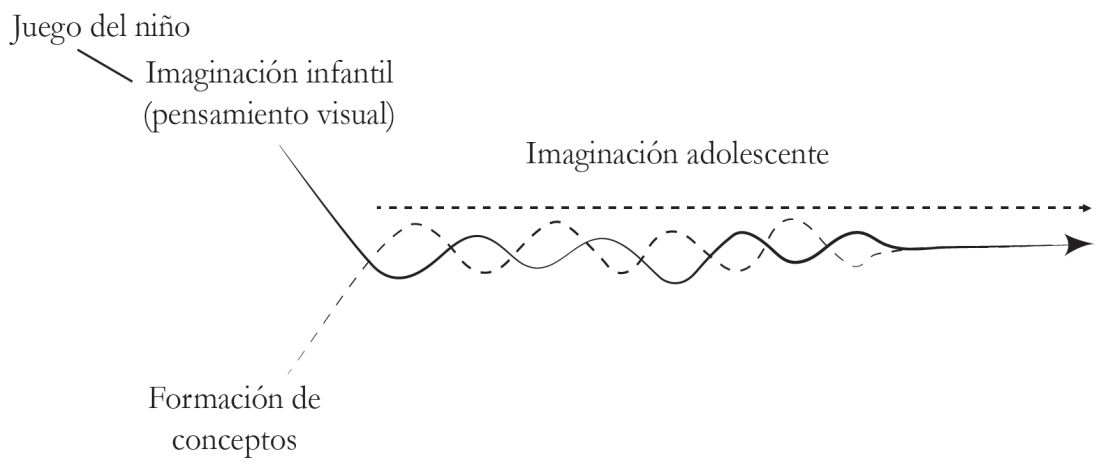

Figura 1. Posible trayectoria genética de la imaginación adolescente. 
juego infantil, sería posible intuir que existe una trayectoria genética que inicia con una actividad social concreta en contextos de interacción específicos: el juego. Este evoluciona reconstructivamente hacia el establecimiento de una función autorregulatoria privada de la esfera emocional, intuición que, a priori, sería compatible con los desarrollos teóricos de Vygotski; por ejemplo, con su caracterización del concepto de habla privada.

Vale la pena notar que, en este texto, la imaginación creativa constituye una unidad de análisis compleja, sistémica, que conjuga las relaciones dialécticas existentes entre el impulso sexual, el juego infantil, el pensamiento en imágenes y el pensamiento mediante verdaderos conceptos. El breve recorrido realizado respecto de la fantasía en el adolescente permite ilustrar el modo en que

(...) sobre la base de la formación de conceptos, se desarrollan algunas funciones mentales completamente nuevas; el modo en que sobre esta nueva base la percepción, la memoria, la atención, y la actividad práctica del adolescente es reestructurada; y lo que es el punto principal, cómo se unen en una nueva estructura, cómo, gradualmente, ciertos cimientos se colocan para habilitar nuevas síntesis superiores de personalidad y visiones del mundo (Vygotski, 1998 [1931], p. 165-166).

\section{Conclusiones y comentarios sobre la relevancia actual de la temática}

En la primera parte de este artículo se propuso un recorrido conceptual por las principales categorías analíticas de los enfoques socioculturales, tanto en su formulación Vygotskiana (Teoría Histórico-Cultural) como en aquellas postvygotskianas. Se señaló, en primer lugar, la importancia de comprender a los PPS como funciones psicológicas humanas con una legalidad diferenciada, originadas en la vida social y que habilitan la creación de consciencia y control autorregulatorio. La diferenciación entre los PPS rudimentarios y avanzados permitió abordar el

${ }^{3}$ Nótese cómo esta afirmación exhibe cierta influencia psicoanalítica freudiana. complejo asunto del surgimiento de los PPS y analizar, muy brevemente, la concepción del desarrollo no teleológico defendida por Vygotski. Finalmente, se abordaron los aportes de la Teoría de la Actividad y de las disquisiciones epistemológico-metodológicas vinculadas con la delimitación de unidades de análisis que permitan estudiar los procesos psicológicos como formas en despliegue longitudinal, y no como muestras fosilizadas de conducta.

En la segunda parte del trabajo, se mostrpo de qué modo aplica Vygotski las categorías de la Teoría Histórico-Cultural al análisis de la imaginación, la creatividad y la fantasía en el adolescente. Se trata de una propuesta analítica ciertamente original y cuya unidad de análisis es verdaderamente compleja: la creatividad se sitúa en el centro de un campo dinámico de relaciones entre pensamiento, emociones, contextos de actividad cotidianos, uso de instrumentos de mediación y procesos de autorregulación.

Además, para Vygotski, la creatividad (como todo proceso psicológico) debe ser abordada longitudinalmente, es decir, como una función en despliegue permanente. Ello tiene implicancias varias, siendo una de las más relevantes la inclusión del tópico de la creatividad al interior de las discusiones sobre el desarrollo psicológico humano. Si, como ya se ha mencionado, existen autores que reniegan de la ausencia de perspectivas que consideren a la creatividad en sus relaciones con la sociedad (Amabile, 1983), es importante reconocer el carácter vanguardista de la mirada histórico-cultural y del desarrollo que Vygotski formuló sobre la creatividad en 1931.

La perspectiva vygotskiana sobre la creatividad no solo reviste valor en tanto aporte históricamente notable, sino que resulta fundamental para cualquier discusión actual de la creatividad. Ello es así porque, dado que para Vygotski “toda función aparece dos veces: primero, a nivel social, y más tarde, a nivel individual" (Vygotski, 1979/2009 [1931-1934], p. 94), las características histórico-culturales del presente resultan centrales para comprender las particularidades y el rol de la creatividad en el mundo contemporáneo. En efecto, el contexto sociocultural de 1931 no es el mismo que el actual. 
La vertiginosa aceleración del ritmo de vida en el trabajo y el hogar, el contexto generalizado de crisis global en relación con los recursos económicos y las identidades culturales, como así también el avance (a veces desmedido) de las nuevas tecnologías son solo algunos de los elementos que configuran el presente. En este escenario hostil, el ser humano asiste a un proceso paradójico: mientras las categorías convencionalmente empleadas para explicar la creatividad están siendo cuestionadas y deconstruidas por el discurso posmoderno (Eisler \& Montuori, 2007), la creatividad, en tanto responsable del avance social, cultural y tecnológico, deviene no tanto una necesidad como una cuestión de supervivencia (Romo Santos, 1997). En palabras de Richards:

La creatividad cotidiana es fundamental para nuestra supervivencia. Gracias a la creatividad cotidiana nos adaptamos flexiblemente, improvisamos y probamos diferentes opciones, tanto si estamos criando a un niño, aconsejando a un amigo, arreglando nuestro hogar, o planeando un evento para recaudar fondos (Richards, 2007, p. 25-26).

En la medida en que la tecnología se hace cargo de la mayor parte de las tareas rutinarias antes realizadas por los humanos, la creatividad se vuelve más y más necesaria, en tanto función psicológica capaz de crear nuevos futuros y empujar a la sociedad a través de procesos de innovación (Moran, 2010; Runco, 2004; Westwood \& Low, 2003). Tal vez por ello, en los últimos años, las discusiones sobre la creatividad y sus posibles aplicaciones en diferentes áreas de la educación se han multiplicado exponencialmente (Hennessey \& Watson, 2016; Santos \& André, 2015; Sintoni, 2016). Convendría entonces, dadas las circunstancias que atraviesan las subjetividades, revalorizar la propuesta teórica vygotskiana y emprender un análisis de la creatividad, la imaginación y la fantasía desde una perspectiva cultural del desarrollo. Ello permitiría, por un lado, ensayar descripciones y explicaciones situadas, socio y ontogenéticamente relevantes, sobre estas funciones psicológicas. Pero, además, habilitaría lecturas más abarcativas, que no desdeñen la complejidad multinivel que la creatividad, la imaginación y la fantasía entrañan, sino que la asuman y la comprendan como condición de posibilidad de ellas.

\section{Referencias}

Amabile, T. (1983). The social psychology of creativity: a componential conceptualization. Journal of Personality and Social Psychology, 45(2), 357-376. doi: 10.1037/0022-3514.45.2.357

Amabile, T. (1988). A model of creativity and innovation in organizations. Research in Organizational Behavior, 10, 123-167.

Atencio, D. J., \& Montero, I. (2009). Private speech and motivation: The role of language in a sociocultural account of motivational processes. In A. Winsler, C. Fernyhough \& I. Montero (Eds.), Private speech, executive functioning, and the development of verbal selfregulation (pp. 201-223). Cambridge: CUP.

Baquero, R. (1996). Vigotsky y el aprendiraje escolar. Buenos Aires: Aique.

Baquero, R. (2001a). Aprendizaje escolar y contexto. En su Introducción a lapsicología del aprendizajeescolar (pp.163199).Buenos Aires:Universidad NacionaldeQuilmes.

Baquero, R. (2001b). La educabilidad bajo sospecha. Cuadernos de Pedagogía, IV(9), 71-85.

Baquero, R. (2009). ZDP, sujeto y situación. El problema de las unidades de análisis en psicología educacional. Actualidades Investigativas en Educación, 9, 1-26.

Baquero, R. (2012a). Vigotsky: sujeto y situación, claves de un programa psicológico. En J. A. Castorina \& M. Carretero (Comps.), Desarrollo Cognitivo y Educación, Vol 1 (pp. 61-86). Buenos Aires: Paidós.

Baquero, R. (2012b). Alcances y límites de la mirada psicoeducativa sobre el aprendizaje escolar: algunos giros y perspectivas. Revista Polifonias, 1(1), 9-21.

Berk, L. E. (1992). Children's private speech: An overview of theory and status of research. In R.M. Días \& L. E. Berk (Eds.), Private speech: From social interaction to self-regulation (pp. 17-53). Hillsdale: Erlbaum. 
Cassirer, E. (1929). Étude sur la pathologie de la conscience symbolique. Journal de Psychologie Normale et Pathologie, 26, 289-336.

Castorina, J. A., \& Baquero, R. (2005). Dialéctica y psicología del desarrollo. El pensamiento de Piaget y Vigotsky. Buenos Aires: Amorrortu.

Cole, M. (1996/1999). Psicología cultural. Una disciplina del pasado y del futuro. Madrid: Morata.

Cubero Pérez, M., \& Santamaría Santiagosa, A. (2005). Psicología cultural: una aproximación conceptual e histórica al encuentro entre mente y cultura. Avances en Psicología Latinoamericana, 23(1), 15-31. doi: 10.12804/revistas.urosario.edu.co/apl/a.1237

Daniels, H. (2001/2003). Vygotsky y la pedagogía. Barcelona: Paidós.

Eisler, R., \& Montuori, A. (2007). Creativity, society, and the hidden subtext of gender: toward a new contextualized approach. World Futures. The Journal of New Paradigm Research, 63(7), 479-499. doi: 10.1080/02604020701572681

Engeström, Y. (2015). Learning by expanding. An activitytheoretical approach to developmental research (2da edición). New York: Cambridge University Press.

Feldman, D. H., Csikszentmihalyi, M., \& Gardner, H. (1994). Changing the world: A framework for the study of creativity. Westport: Praeger Publishers/Greenwood Publishing Group.

Fernyhough, C. (2004). Alien voices and inner dialogue: towards a developmental account of auditory verbal hallucinations. New Ideas in Psychology, 22(1), 49-68. doi: 10.1016/j.newideapsych.2004.09.001

Fernyhough, C., \& Fradley, E. (2005). Private speech on an executive task: relations with task difficulty and task performance. Cognitive Development, 20(1), 103-120. doi: 10.1016/j.cogdev.2004.11.002

Glăveanu, V. P. (2010). Paradigms in the study of creativity: Introducing the perspective of cultural psychology. New Ideas in Psychology, 28(1), 79-93. doi: 10.1016/j.newideapsych.2009.07.007
Hennessey, B. A., \& Watson, M. W. (2016). The defragmentation of creativity: future directions with an emphasis on educational applications. In G. E. Corazza y S. Agnoli (Eds.) Multidisciplinary contributions to the science of creative thinking (pp. 2132). Singapore / Heidelberg: Springer.

Kim, M. (2016). Importance of creative thinking for paradigm shifts that foster scientific advances. In M. K. Demetrikopoulos y J. L. Pecore (Eds.), Interplay of creativity and giftedness in science (pp. 15-28). Rotterdam / Boston: Sense Publishers.

Leontiev, A. N. (1972/1977). Activity and consciousness. En VV.AA., Philosophy in the USSR. Problems of dialectical materialism (pp. 180-202). Moscow: Progress Publishers.

Lewin, K. (1931). The conflict between Aristotelian and Galilean modes of thought in contemporary psychology. The Journal of General Psychology, 5(2), 141-177. doi: 10.1080/00221309.1931.9918387

Maier, N. R. F. (1970). Problem solving and creativity. In individuals and groups. Belmont: Brooks/Cole Publishing Co.

Montero, I. \& de Dios, M. J. (2006). Vygotsky was right. An experimental approach to the study of the relationship between private speech and task performance. Estudios de Psicología, 27(2), 175-189.

Moran, S. (2010). The roles of creativity in society. In J. C. Kaufman, \& R. J. Sternberg (Eds.), The Cambridge bandbook of creativity (pp. 74-92). Cambridge / New York: Cambridge University Press.

Paulus, P., \& Nijstad, B. (2003). Group creativity: an introduction. In P. Paulus, \& B. Nijstad (Eds.), Group creativity: Innovation through collaboration (pp. 3-11). New York: Oxford University Press.

Piaget, J. (1936/1981). El nacimiento de la inteligencia en el niño. Buenos Aires: Ábaco.

Richards, R. (2007). Everyday creativity: Our hidden potential. In R. Richards (Ed.), Everyday creativity and new views of buman nature. Psychological, social, and spiritual perspectives (pp. 25-54). Washington: American Psychological Association. 
Rivière, Á. (1988). La psicología de Vigotski. Madrid: Aprendizaje Visor.

Rivière, Á. (2002). Desarrollo y educación: el papel de la educación en el «diseño» del desarrollo humano. Obras Escogidas Vol. III (pp. 203-242). Madrid: Editorial Médica Panamericana.

Rogoff, B. (1997). Los tres planos de la actividad sociocultural: apropiación participativa, participación guiada y aprendizaje. En J. Wertsch, P. Del Río, \& A. Álvarez (Eds.), La mente sociocultural. Aproximaciones teóricas y aplicadas. Madrid: Fundación Infancia y Aprendizaje.

Romo Santos, M. (1997). Psicología de la creatividad. Barcelona / Buenos Aires: Paidós.

Runco, M. (2004). Creativity. Annual Review of Psychology, 55, 657-687. doi: 10.1146/annurev. psych.55.090902.141502

Sánchez-Ruiz, M. J., Romo Santos, M., \& Jiménez Jiménez, J. (2013). The role of metaphorical thinking in the creativity of scientific discourse. Creativity Research Journal, 25(4), 361-368. doi: 10.1080/10400419.2013.843316

Santos, M. T., \& André, M. (2015). Conceções de educadores de infância sobre criatividade. Investigar em Educaşão, II(4), 97-112.

Sintoni, C. (2016). Music listening, composition and performance: An experience of creativity for education. In G. E. Corazza \& S. Agnoli (Eds.) Multidisciplinarycontributionstothescience of creativethinking (pp. 301-324). Singapore / Heidelberg: Springer.

Sternberg, R. J. (1999). Handbook of creativity. New York: Cambridge University Press.

Stuart-Mill, J. (2011 [1843]). A system of logic. Ratiocinative and inductive, being a connected view of the principles of evidence, and the methods of scientific investigation. South Australia: University of Adelaide.

Valsiner, J. (2000). Culture and buman development: An introduction. LondonThousand Oaks, CA: SAGE Publications Ltd.
Valsiner, J. (2007). Culture in minds and societies: Foundations of cultural psychology. New Delhi: SAGE Publications India Pvt. Ltd.

Valsiner, J. (2012). La dialéctica en el estudio del desarrollo. En J. A. Castorina, \& M. Carretero (Eds.), Desarrollo cognitivo y educación [1]. Los inicios del conocimiento (p. 137-164). Buenos Aires: Paidós.

Vygotski, L. S. (1973/1987[1934]). Pensamiento y lenguaje (2da edición). Buenos Aires: La Pléyade.

Vygotski, L. S. (1979/2009 [1931-1934]). El desarrollo de los procesos psicológicos superiores. Buenos Aires: Crítica.

Vygotsky, L. S. (1998). The collected works of L.S. Vygotsky (1896-1934). Volume 5: Child psychology (R. W. Rieber, Ed.; S. Sochinenii, Trad.). New York: Springer Science + Business Media.

Weiner, R. P. (2000). Creativity and beyond: Cultures, values, and change. Albany: State University of New York Press.

Weisberg, R. W. (2006). Creativity. New Jersey: John Wiley \& Sons.

Wertsch, J. V. (1991/1993). Voices of the mind. A sociocultural approach to mediated action. Cambridge: Harvard University Press.

Wertsch, J. V. (1998). Mind as action. New York: Oxford University Press.

Westwood, R., \& Low, D. (2003). The multicultural muse: culture, creativity and innovation. International Management of Cross-Cultural Management, 3(2), 235259. doi: $10.1177 / 14705958030032006$

Winsler, A. (2009). Still talking to ourselves after all these years: A review of current research on private speech. In A. Winsler, C. Fernyhough e I. Montero (Eds.), Private speech, executive functioning, and the development of verbal self-regulation (pp. 3-41). Cambridge: CUP.

Winsler, A., Díaz, R., \& Montero, I. (1997). The role of private speech in the transition from collaborative to independent task performance in young children. Early Childhood Research Quarterly, 12(1), 59-79. doi: 10.1016/S0885-2006(97)90043-0 
Wundt, W. (1916/2003 [1904]). Elements of folk psychology: Outlines of a psychological history of the development of mankind. New York, USA: Routledge.
Yaroshevsky, M. (1989). Lev Vygostky. Moscow: Progress Publishers.

Recibido: 11 de noviembre de 2016

Aceptado: 29 de mayo de 2017 